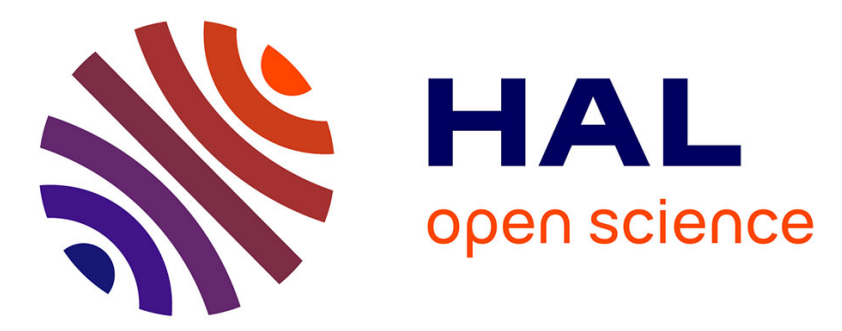

\title{
Robust relay control for buck converters : experimental application
}

Aboubacar Ndoye, Romain Delpoux, Laurentiu Hetel, Alexandre Kruszewski, Jean-François Trégouët, Xuefang Lin-Shi

\section{- To cite this version:}

Aboubacar Ndoye, Romain Delpoux, Laurentiu Hetel, Alexandre Kruszewski, Jean-François Trégouët, et al.. Robust relay control for buck converters: experimental application. Conference on Decision and Control, Dec 2019, Nice, France. 10.1109/CDC40024.2019.9030179 . hal-02344388v2

\section{HAL Id: hal-02344388 \\ https://hal.science/hal-02344388v2}

Submitted on 7 Nov 2019

HAL is a multi-disciplinary open access archive for the deposit and dissemination of scientific research documents, whether they are published or not. The documents may come from teaching and research institutions in France or abroad, or from public or private research centers.
L'archive ouverte pluridisciplinaire HAL, est destinée au dépôt et à la diffusion de documents scientifiques de niveau recherche, publiés ou non, émanant des établissements d'enseignement et de recherche français ou étrangers, des laboratoires publics ou privés. 


\title{
Robust relay control for buck converters : experimental application
}

\author{
A. Ndoye ${ }^{1}$, R. Delpoux ${ }^{1}$, L. Hetel ${ }^{2}$, A. Kruszewski ${ }^{3}$, J.-F. Trégouët ${ }^{1}$ and X. Lin-Shi ${ }^{1}$
}

\begin{abstract}
In this paper, a robust relay control of a buck converter is proposed. The controller is synthesized based on the design of a continuous controller for the average model of a buck converter usually used when Pulse Width Modulation (PWM) is considered. To deal with parameter uncertainties, the controller implements an integral action. In this paper, local stability is proven for the hybrid system, thereby ensuring zero steady state error on the voltage output. A subset of the robust basin of attraction is explicitly constructed. The effectiveness of this control is illustrated experimentally.
\end{abstract}

\section{INTRODUCTION}

DC-DC converters are devices which are used in many modern applications including power systems and power electronics [13], due to their high frequency, low cost and small size [5]. They belong to the class of switched system which represents the subclass of hybrid systems for which active subsystem is selected according to some switching rule [14]. The study of switched system has attracted a lot of attention from the scientific community over the past few years [4] [9] [10]. In the literature, we find mainly two approaches for DC-DC converters control. The most widely used approach in practice is based on the use of Pulse Width Modulation (PWM) [8]. In this approach the hybrid character of the system is bypassed considering an average model for converters. The average model assumption is validated for large switching frequency. The method is simple to implement and has the advantage to have a constant and known sampling frequency. However, it is necessary to maintain a frequency separation between the closed loop system dynamic and the switching frequency. On the other hand relay control, where the control takes value in a finite set of constant vectors has been widely studied. However, few practical implementations are proposed. There are two main reasons for this. In the literature, robustness of the control is of particular interest. Indeed, many contributions are dedicated to the design of stabilizing rules for the case where the equilibrium point is known. Nevertheless, they cannot be applied in the practical case where uncertainties may appear. Besides, this approach requires a great computing capacity.

This work was supported in part by ANR project DIGITSLID (ANR-18CE40-000).

${ }^{1}$ A. Ndoye, R. Delpoux, J.-F. Trégouët and X. Lin-Shi are with UMR 5005 - Ampère Lab, INSA LYON, Université de Lyon, 20, Avenue Albert Einstein, 69100 Villeurbanne, France

${ }^{2}$ L. Hetel is with Univ. Lille, CNRS, Centrale Lille, UMR 9189 CRIStAL - Centre de Recherche en Informatique Signal et Automatique de Lille, F-59000 Lille, France.

${ }^{3}$ A. Kruszewski belongs to Defrost team, Inria, Univ. Lille, Centrale Lille, UMR 9189 - CRIStAL - Centre de Recherche en Informatique Signal et Automatique de Lille, France.
In this paper, a new approach for the design of a relay control for buck converters is presented. The proposed approach is able to deal with uncertain parameters. The controller is synthesized based on the design of a continuous controller for the average model of a buck converter usually used when PWM is considered. In this paper the results of [7] are revised to cope with time-varying parameter such as a load variation by implementing an integral action. Local stability is proven, thereby ensuring zero steady state error on the voltage output in a hybrid framework. A similar methodology can be found in [1]. With the use of an integral action, the authors guarantee zero steady state error under the assumption that the discrepancy in the modelling is bounded. However, the limits of this discrepancy are not explicitly defined. In this paper, we guarantee the existence of an equilibrium for which the reference of the output is always achieved for all unknown parameters which are supposed to belong to a bounded known interval by using a polytopic model. Besides a subset included in the robust basin of attraction is explicitly constructed. Finally, the evolution of technology and computing resources makes it possible to implement experimentally the proposed approach. The uses of a user-programmable Field-Programmable Gate Array (FPGA) demonstrates the effectiveness of the proposed approach experimentally.

The paper is structured as follows: Section II presents the modelling of the buck converter which will be used in this paper and the problem formulation. Section III is dedicated to the main contribution of this paper. It is about, first, designing the robust relay control from the existence of a continuous control, then we present a constructive method for designing a subset included in the robust domain of attraction. In Section IV, the control is applied to a buck converter, the effectiveness of the control is presented through simulation and experimental results.

Notations: By $|\cdot|$, we denote the Euclidean vector norm of a vector. For a matrix $M, M^{\top}$ denotes the transpose of $M . B\left(x, c_{B}\right)$ denotes the open ball centered on $x$ with radius $c_{B}>0: B\left(x, c_{B}\right)=\left\{y \in \mathbb{R}^{n}:|x-y|<c_{B}\right\}$ and $\mathcal{I}_{N}$ represents the set $\{1,2, \ldots, N\}$. For a symmetric positive definite matrix $P \in \mathbb{R}^{n \times n}$ and a positive scalar $c$, we denote by $\mathcal{E}(P, c)$ the ellipsoid $:\left\{x \in \mathbb{R}^{n}: x^{T} P x<c\right\}$. The convex hull of the set $\mathcal{U}$ is denoted by $\overline{\operatorname{conv}}\{\mathcal{U}\}$.

\section{Problem Statement}

The circuit diagram of the buck converter is shown in Fig. 1. This converter belongs to the class of "chopper" circuits or attenuation circuits [12], since output voltage magnitude is lower than the input one. 


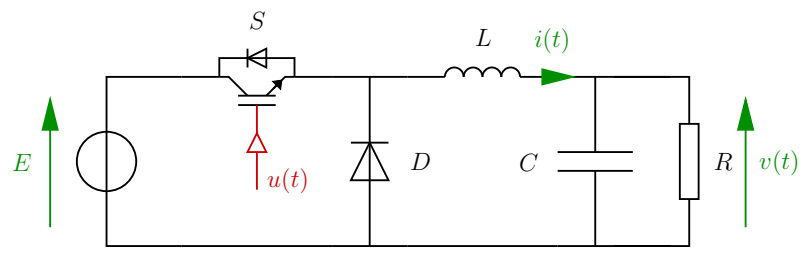

Fig. 1: Electrical scheme

The inductor current and the load voltage are noted $i$ and $v$ respectively. The resistor $R$ models the converter load, while $C$ and $L$ represent the capacitance and the inductance respectively. The parameter $E$ is the magnitude of the voltage source. In this article we consider the ideal topology: parasitic elements of the electrical components are dismissed and the transistor $\mathrm{S}$ as well as the diode $D$ are replaced by switch and wire as shown in Fig. 2.

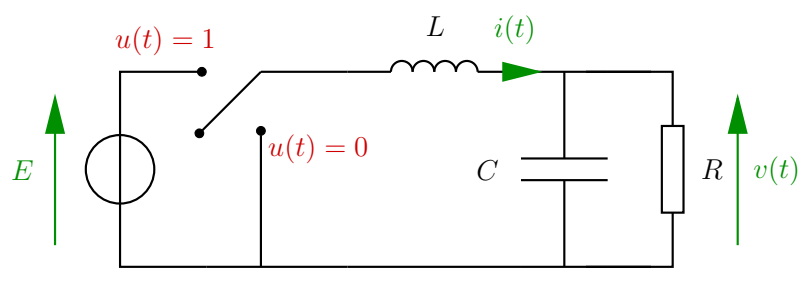

Fig. 2: Ideal representation of the buck converter

With the state vector $x=[i, v]^{\top}$, the dynamical model of the buck converter is represented by the following equations:

$$
\dot{x}=\tilde{A}_{j} x+\tilde{B}_{j}, \quad j \in\{1,2\}
$$

where

$$
\begin{gathered}
\tilde{A}_{1}=\left[\begin{array}{cc}
0 & \frac{-1}{L} \\
\frac{1}{C} & -\frac{1}{R C}
\end{array}\right], \quad \tilde{B}_{1}=\left[\begin{array}{c}
\frac{E}{L} \\
0
\end{array}\right] \\
\tilde{A}_{2}=\left[\begin{array}{cc}
0 & \frac{-1}{L} \\
\frac{1}{C} & -\frac{1}{R C}
\end{array}\right], \quad \tilde{B}_{2}=\left[\begin{array}{l}
0 \\
0
\end{array}\right]
\end{gathered}
$$

Besides, considering the fact that $\tilde{A}_{1}=\tilde{A}_{2}$ and $\tilde{B}_{2}=0_{2 \times 1}$, we obtain a simpler representation

$$
\dot{x}=\tilde{A}_{1} x+\tilde{B}_{1} u,
$$

where the control $u$ governs the switch and takes value in the set $\mathcal{U}$ defined as follows:

$$
\mathcal{U}=\{0,1\}
$$

For this converter, the control aims regulating the output voltage $v(t)$ to a given level $V_{\text {ref }}$ for all unknown parameter which are supposed to belong to a bounded known interval. Due to uncertainties a steady state error may occur. For this purpose the control law implements an integral action.

Model (4) is augmented with an additional state variable $z$ such as $z=\int\left(x_{2}-V_{\text {ref }}\right)$ which is the integral of the output voltage error. Thus by denoting $\zeta=\left[\begin{array}{ll}x^{\top} & z\end{array}\right]^{\top}$, the augmented model is written as:

$$
\dot{\zeta}=A(\theta) \zeta+B u+h
$$

where $\zeta \in \mathbb{R}^{3}, \theta:=1 / R$ with $\theta \in \Theta:=\left[\theta_{\text {min }}, \theta_{\text {max }}\right]$, with

$$
A(\theta)=\left[\begin{array}{ccc}
0 & \frac{-1}{L} & 0 \\
\frac{1}{C} & -\frac{1}{C} \cdot \theta & 0 \\
0 & 1 & 0
\end{array}\right], B=\left[\begin{array}{c}
\frac{E}{L} \\
0 \\
0
\end{array}\right], \quad h=\left[\begin{array}{c}
0 \\
0 \\
-V_{\text {ref }}
\end{array}\right]
$$

Due to the discontinuities of the control $u \in \mathcal{U}$, the solutions of the closed-loop will be considered in the sense of Fillipov [6].

Remark 1: Because of the integral action, the matrix $A(\theta)$ is not full rank. Therefore, classical approaches [3] [2] for the global stabilization of a switched system to an equilibrium point cannot be used directly. However our approach allows to avoid this obstruction by restricting to local stabilization : the switching law is designed by embedding locally the behaviour of a continuous controller.

Remark 2: The matrix $A(\theta)$ is affine with respect to the parameter $\theta$. So it is possible to define a convex polytope of $N=2$ vertices containing all the admissible values of the uncertain matrix. The vertices of this polytopic model are defined as follows:

$$
A_{1}=A\left(\theta_{\min }\right) ; \quad A_{2}=A\left(\theta_{\max }\right)
$$

These vertices will be used in the next section in order to design a robust relay control for the buck converter.

\section{UNCERTAIN RELAY CONTROL SYNTHESIS}

\section{A. State feedback stabilization}

In the continuous framework, control synthesis for uncertain parameters with integral action has already been widely treated in the literature, see for example [11]. Following this strategy, let us first define the set $\Phi_{e}$ of equilibria of Filippov solutions of (6) for $u \in \overline{\operatorname{conv}}\{\mathcal{U}\}$, that is:

$$
\Phi_{e}=\left\{\left(\zeta_{\theta}^{*}, u_{\theta}^{*}\right) \in \mathbb{R}^{3} \times[0,1]: A(\theta) \zeta_{\theta}^{*}+B u_{\theta}^{*}+h=0\right\}
$$

From the definition of matrices in (7), resolving equation involved in (9) leads to:

$$
\left\{\begin{array}{l}
\frac{-1}{L} v_{\theta}^{*}+\frac{E}{L} u_{\theta}^{*}=0 \\
\frac{1}{C} i_{\theta}^{*}-\frac{1}{R C} v_{\theta}^{*}=0 \\
v_{\theta}^{*}=V_{\text {ref }}
\end{array}\right.
$$

So that:

$$
\left\{\begin{array}{l}
i_{\theta}^{*}=\theta \cdot V_{\text {ref }}, \\
v_{\theta}^{*}=V_{\text {ref }}, \\
u_{\theta}^{*}=\frac{V_{\text {ref }}}{E},
\end{array}, \forall z_{\theta}^{*} \in \mathbb{R} .\right.
$$

As a result, $\Phi_{e}$ admits the following explicit expression:

$$
\Phi_{e}=\left[\begin{array}{l}
\theta \\
1
\end{array}\right] V_{\text {ref }} \times \mathbb{R} \times\left\{\frac{V_{r e f}}{E}\right\}
$$


Note that the introduction of the integral action eliminates the $\theta$ dependency on the voltage output at the equilibrium.

Let us state the main result of the article.

Theorem 1: Given any $\theta_{n} \in \Theta$ and $\left(\zeta_{\theta_{n}}^{*}, u_{\theta_{n}}^{*}\right) \in \Phi_{e}$ with:

$$
z_{\theta_{n}}^{*}=0
$$

Assume that there exist $P=P^{\top} \in \mathbb{R}^{3 \times 3}$ and $K \in \mathbb{R}^{1 \times 3}$ such that:

$$
\begin{gathered}
P=\left(\begin{array}{ccc}
p_{1} & p_{1,2} & p_{1,3} \\
* & p_{2} & p_{2,3} \\
* & * & p_{3}
\end{array}\right) \succ 0 \\
p_{1,3} \neq 0 \\
(A(\theta)+B K)^{\top} P+P(A(\theta)+B K)+\delta P \prec 0, \forall \theta \in \Theta .
\end{gathered}
$$

Then, there exists $\left(\zeta_{\theta}^{*}, u_{\theta}^{*}\right) \in \Phi_{e}$ and positive scalars $\mathcal{C}, \epsilon$ with

$$
z_{\theta}^{*}=-\frac{p_{1}\left(\theta-\theta_{n}\right) V_{r e f}}{p_{1,3}}
$$

such that the Filippov solutions $\zeta(t)$ of the closed-loop system (6), under the control law:

$u(\zeta)=1-\operatorname{sign}\left(\left(\zeta-\zeta_{\theta_{n}}^{*}\right)^{\top} P B\right)=\left\{\begin{array}{l}0,\left(\zeta-\zeta_{\theta_{n}}^{*}\right)^{\top} P B \geq 0 \\ 1,\left(\zeta-\zeta_{\theta_{n}}^{*}\right)^{\top} P B<0\end{array}\right.$

satisfy

$$
\left\|\zeta(t)-\zeta_{\theta}^{*}\right\|^{2} \leq \mathcal{C} e^{-\delta t}\left\|\zeta(0)-\zeta_{\theta}^{*}\right\|^{2}, \forall t \geq 0 .
$$

for all $\left\|\zeta(0)-\zeta_{\theta}^{*}\right\|^{2}<\epsilon$.

Proof: The proof uses arguments based on results provided by [7]. The control synthesis is based on the existence of linear state feedback with gain $K$.

Consider the coordinates transformation $\eta=\zeta-\zeta_{\theta}^{*}, v=$ $u-u_{\theta}^{*}$. System (6) can be re-written as

$$
\dot{\eta}=A(\theta) \eta+B v
$$

where relation (9) has been used. Note that $v$ takes values in the set $\mathcal{V}$ defined as follows:

$$
\mathcal{V}=\left\{-u_{\theta}^{*}, 1-u_{\theta}^{*}\right\}
$$

with $u_{\theta}^{*}=V_{\text {ref }} / E$.

Then from (16), $V(\eta)=\eta^{\top} P \eta$ satisfies:

$$
\frac{\partial V}{\partial \eta}(A(\theta) \eta+B K) \eta<-\delta V(\eta), \forall \eta \neq 0 .
$$

Let us denote:

$$
C_{\mathcal{V}}(K):=\left\{x \in \mathbb{R}^{n}: K x \in \overline{\operatorname{conv}}(\mathcal{V})\right\}
$$

Since $0 \in \operatorname{Int}\{\overline{\operatorname{conv}}(\mathcal{V})\}=]-u_{\theta}^{*}, 1-u_{\theta}^{*}\left[\right.$ for any $V_{\text {ref }} \neq 0$, there exits a level set such that:

$$
\Omega_{0}:=\mathcal{E}(P, 1) \subset C_{\mathcal{V}}(K)
$$

Note that for any $\eta \in \Omega_{0}$, there exits $N$ scalars $\alpha_{j}(\eta) \geqslant$ $0, \forall j \in I_{N}$, with $\sum_{j=1}^{N} \alpha_{j}(\eta)=1$ such that:

$$
K \eta=\sum_{j=1}^{N} \alpha_{j}(\eta) v_{j}
$$

From (22) and (25), we have:

$$
\sum_{j=1}^{N} \alpha_{j}(\eta) \frac{\partial V}{\partial \eta}\left(A(\theta) \eta+B v_{j}\right)<-2 \delta V(\eta), \forall \eta \in \Omega_{0} \backslash\{0\}
$$

Now considering that $\alpha_{j}(\eta) \geqslant 0, \forall j \in I_{N}$, there must be at least one $j \in I_{N}$ such that:

$$
\frac{\partial V}{\partial \eta}\left(A(\theta) \eta+B v_{j}\right)<-\delta V(\eta), \forall \eta \in \Omega_{0} \backslash\{0\}
$$

Thus by choosing the control $v$ such that

$$
v(\eta) \in \arg \min _{\tilde{v} \in \mathcal{V}} \eta^{T} P B \tilde{v}
$$

one ensures the existence of positive scalars $\mathcal{C}$ and $\epsilon$ such that the Filippov solutions $\eta(t)$ of system (20), (28) with $\|\eta(0)\|^{2}<\epsilon$, satisfy

$$
\|\eta(t)\|^{2} \leq \mathcal{C} e^{-\delta t}\|\eta(0)\|^{2}, \forall t \geq 0 .
$$

For $\mathcal{V}=\left\{-u_{\theta}^{*}, 1-u_{\theta}^{*}\right\}$, the control

$$
v(\eta)= \begin{cases}-u_{\theta}^{*}, & \text { if } B^{\top} P \eta \geq 0 \\ 1-u_{\theta}^{*}, & \text { if } B^{\top} P \eta<0\end{cases}
$$

is a particular case of (28). By definition, $v=u-u_{\theta}^{*}$ and $\eta=\zeta-\zeta_{\theta}^{*}$, that is, the control applied to system (6) takes the form

$$
u(\zeta)= \begin{cases}0, & \text { if } B^{\top} P\left(\zeta-\zeta_{\theta}^{*}\right) \geq 0 \\ 1, & \text { if } B^{\top} P\left(\zeta-\zeta_{\theta}^{*}\right)<0\end{cases}
$$

Note that $\zeta_{\theta}^{*}$ is unknown, then we can re-expressed $B^{T} P(\zeta-$ $\left.\zeta_{\theta}^{*}\right)$ as:

$$
B^{\top} P\left(\zeta-\zeta_{\theta}^{*}\right)=B^{\top} P\left(\zeta-\zeta_{\theta_{n}}^{*}\right)-B^{\top} P\left(\zeta_{\theta}^{*}-\zeta_{\theta_{n}}^{*}\right)
$$

From (13) and (17)

$$
\begin{aligned}
& B^{\top} P\left(\zeta_{\theta}^{*}-\zeta_{\theta_{n}}^{*}\right)= \\
& \left(\begin{array}{lll}
E / L & 0 & 0
\end{array}\right)\left(\begin{array}{ccc}
p_{1} & p_{1,2} & p_{1,3} \\
* & p_{2} & p_{2,3} \\
* & * & p_{3}
\end{array}\right)\left(\begin{array}{c}
\left(\theta-\theta_{n}\right) V_{\text {ref }} \\
0 \\
-\frac{p_{1}\left(\theta-\theta_{n}\right) V_{\text {ref }}}{p_{1,3}}
\end{array}\right) \\
& =0 .
\end{aligned}
$$

Since $B^{\top} P \zeta_{\theta}^{*}=B^{\top} P \zeta_{\theta_{n}}^{*}$, control (31) can be re-expressed as

$$
u(\zeta)= \begin{cases}0, & \text { if } B^{\top} P\left(\zeta-\zeta_{\theta_{n}}^{*}\right) \geq 0 \\ 1, & \text { if } B^{\top} P\left(\zeta-\zeta_{\theta_{n}}^{*}\right)<0\end{cases}
$$

which is the same as control (18).

From (29), using the definition of $\eta=\zeta-\zeta_{\theta}^{*}$, the relation (19) holds, which ends the proof.

\section{B. Constructive method for the control synthesis}

Here we design a subset included in the robust domain of attraction. The subset is represented by ellipsoid $\mathcal{E}(P, 1)$. This guarantees that any solution of system (20) originating from the invariant ellipsoid is exponentially converging to the origin with the decay rate $\delta$ for any $\theta \in \Theta$. The methodology that we present here is adapted from [7]. Roughly speaking, it is about optimizing the size of this ellipsoid, using LMI. 
However, to obtain a LMI formulation, the problem could be boiled down to optimize the size of a ball with radius $c_{B}>0$ included in the ellipsoid.

Remark that the set $\overline{\operatorname{conv}}(\mathcal{V})$ used in (23), is a convex polytope, so it can be described by a finite number $N_{g}$ of vectors $g_{i} \in \mathbb{R}^{m}$ such that [15]:

$$
\overline{\operatorname{conv}}(\mathcal{V})=\left\{v \in \mathbb{R}^{m}: g_{i}^{\top} v \leqslant 1, i \in \mathcal{I}_{N_{g}}\right\}
$$

Lemma 1: Consider system (20). For $\epsilon=1 / c_{B}^{2}$ and for a given decay rate $\delta>0$, let $P=Q^{-1}$ and $\lambda$ represent the solutions of the following LMI optimization problem:

$$
\begin{gathered}
\inf _{\epsilon} \quad \text { s.t. } \\
Q=Q^{\top}>0, \lambda>0 ; \\
A_{1} Q+Q A_{1}^{\top}-\lambda B B^{\top}<-2 \delta Q ; \\
A_{2} Q+Q A_{2}^{\top}-\lambda B B^{\top}<-2 \delta Q ; \\
{\left[\begin{array}{cc}
\epsilon I & I \\
I & Q
\end{array}\right]>0} \\
\frac{\lambda}{2} g_{k} B^{\top} \\
{\left[\begin{array}{cc}
1 \\
\left(\frac{\lambda}{2} g_{k} B^{\top}\right)^{\top}
\end{array}\right]>0, \text { with } k \in \mathcal{I}_{N_{g}} .}
\end{gathered}
$$

If $p_{1,3} \neq 0$ as specified in (15), then any solution of system (20) under the control law (28) with $P=Q^{-1}$ originating from the invariant ellipsoid $\mathcal{E}(P, 1)$ is exponentially converging to the origin with the decay rate $\delta$.

Proof: The set of LMIs (35b),(35c) guarantees that the function $V(\eta)=\eta^{\top} P \eta$ satisfies the relation (22) with $K=\frac{-\lambda}{2} B^{\top} Q^{-1}$.

The LMI (35d) ensures that $B\left(0, c_{B}\right) \subset \mathcal{E}(P, 1)$.

And finally the set of LMIs (35e), by applying the Schur complement, guarantees that:

$$
1-g_{k}^{\top} K Q K^{\top} g_{k}>0, k \in \mathcal{I}_{N_{g}}
$$

By remarking that the minimum of $V$ along the hyperplane $g_{k}^{\top} K \eta=1$ is given by $\min _{g_{k}^{\top} K \eta=1} \eta^{\top} P \eta=\left(g_{k}^{\top} K Q K^{\top} g_{k}\right)^{-1}$, then (36) ensures that for any $\eta \in \mathcal{E}(P, 1)$, we have $g_{k}^{\top} K \eta \leqslant$ $1, k \in \mathcal{I}_{N_{g}}$, i.e. $K \eta \in \overline{\operatorname{conv}}(\mathcal{V})$ which guarantees that the set $\mathcal{E}(P, 1)$ is in the domain of attraction of the equilibrium point $\eta=0$.

\section{Simulation AND EXPERIMENTAL RESUlts}

\section{A. Experimental setup presentation}

The test bench used to illustrate the proposed control law is represented in Fig. 3. The buck converter parameters are shown in Table I.

TABLE I: Buck converter parameters

\begin{tabular}{c|c}
\hline Parameters & Values \\
\hline \hline$R$ & {$[5,10] \Omega$} \\
$E$ & $24 \mathrm{~V}$ \\
$C$ & $40 \mu \mathrm{F}$ \\
$L$ & $1.3 \mathrm{mH}$ \\
\hline
\end{tabular}

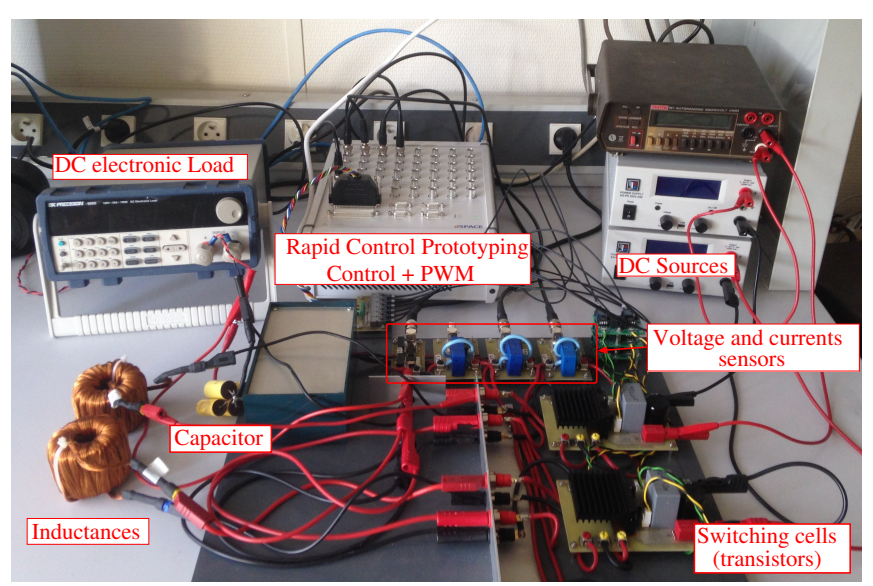

Fig. 3: Test bench

The controller hardware is Rapid Control Prototyping (RCP) system dSpace MicroLabBox which is composed of a Real-Time Processor (RTP) that communicates with a userprogrammable FPGA and several (digital and analog) inputs/outputs. The RTP (NXP QorIQ P5020, dual-core 2GHz) operates with a sampling frequency $T_{s, \text { RTP }}=1.10^{-4} s$. It provides a human machine interface and delivers control

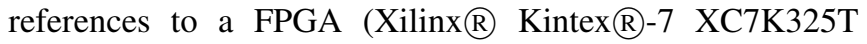
FPGA) implementing the control law. The FPGA clock has a period of $1.10^{-8} \mathrm{~s}$. Experimentally, the MOSFET switching causes the appearance of transitory phenomena on variables to be measured. For this reason, the ADC sampling needs to be triggered with the maximum switching frequency so that measurements are interspersed between two switches. Knowing that on the acquisition system, a measurement takes $1.10^{-6} s$, and that both measurements can be parallelized, the minimum time between two variations of the control can be fixed at $T_{s, \text { FPGA }}=5 \cdot 10^{-6} \mathrm{~s}$. A counter, implemented for this purpose, ensures a constant shift between the switching instants and the measurements. The proposed mechanism is represented in Fig. 4 where we have set the value of the sampling period to $T_{s, \mathrm{FPGA}}=5 \mu \mathrm{s}$ for tests. Therefore the update period for the control is offset from a time equals to $T_{s, \mathrm{FPGA}} / 2=2.5 \mu \mathrm{s}$.

\section{B. Results}

Consider the system (6). For the fixed target value $V_{\text {ref }}=$ $18 \mathrm{~V}$, the set $\mathcal{V}=\{-0.75,0.25\}$ and $\zeta_{\theta_{n}}^{*}=\left(\begin{array}{lll}1.8 & 18 & 0\end{array}\right)^{\top}$. The set $\overline{\operatorname{conv}}(\mathcal{V})$ described in (34) is characterized by $g_{1}=$ $-1.33, g_{2}=4$. For $\delta=1300$, the optimization problem (35) leads to a control law of the form (18) with:

$$
P=\left(\begin{array}{ccc}
0.1 & 7.11 .10^{-4} & 73 \\
7.11 .10^{-4} & 3.34 .10^{-4} & 0.95 \\
73 & 0.95 & 5.74 .10^{3}
\end{array}\right)
$$

which ensures the local stabilization in the ellipsoid $\mathcal{E}(P, 1)$. For the results presented in the sequel, the initial value of the load is, first, fixed to his nominal value equals to $10 \Omega$. Then a load step is applied, which changes the value to $5 \Omega$. The new equilibrium point is therefore equal to : $\zeta_{\theta}^{*}=\left(\begin{array}{lll}3.6 & 18-\end{array}\right.$ 

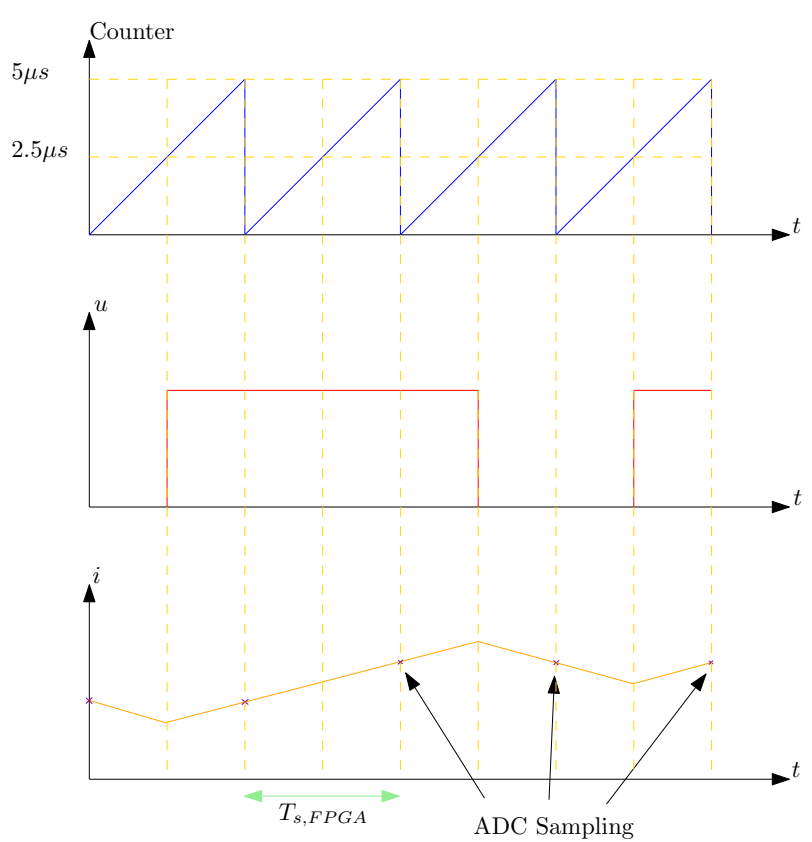

Fig. 4: ADC sampling synchronization

$0.0025)^{\top}$. Thereafter, we show the robustness of the control facing this perturbation.

Remark 3: The matrix $P$ is obtained by solving the optimization problem (18). Therefore, its coefficients depend on the buck converter parameters which are involved in the matrix $A_{1}, A_{2}$ and $B$. This justifies the values obtained.
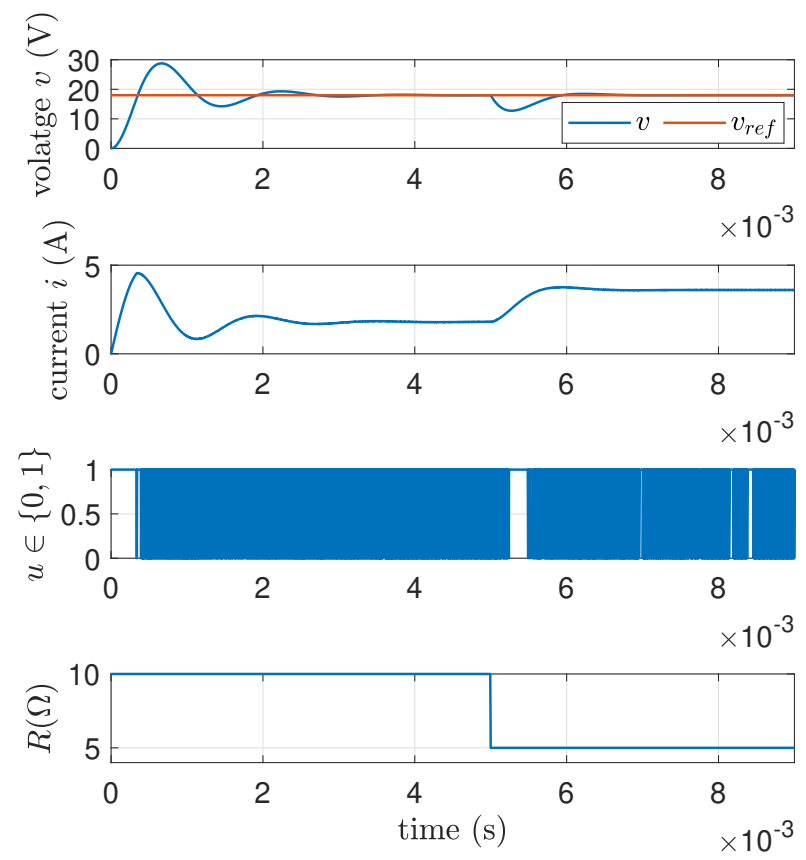

Fig. 5: Load and line transient
1) Simulation results: The simulation results are depicted in Fig. 5 and Fig. 6. Firstly, with the nominal parameter which are known, we can see that the control is able to regulate the state to the reference $V_{\text {ref }}=18 \mathrm{~V}$. Once the load variation occurs, the system remains stable and the control is able to reach the new equilibrium point $\zeta_{\theta}^{*}$ with zero steady error in the controlled output. Note that it is possible to change the dynamics of the system by modifying the parameter $\delta$.
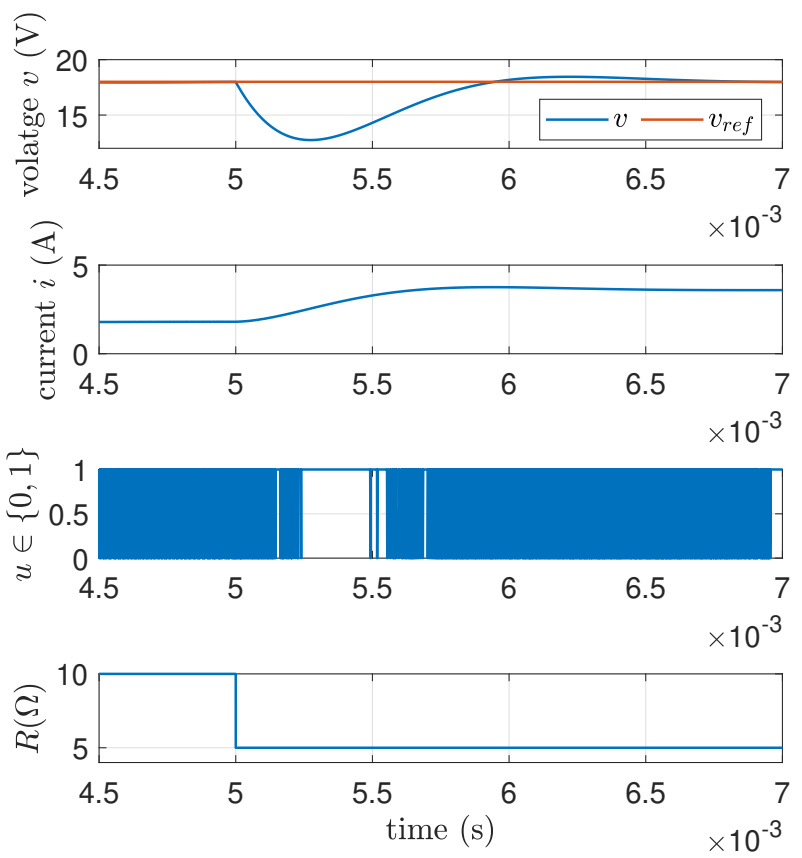

Fig. 6: Zoom of the figure 5

2) Experimental results: To prove the effectiveness of this approach, the target value is changed to $V_{\text {ref }}=12 \mathrm{~V}$ for the experimental tests. Therefore the set $\mathcal{V}=\{-0.5,0.5\}$ and $\zeta_{\theta_{n}}^{*}=\left(\begin{array}{lll}1.2 & 12 & 0\end{array}\right)^{\top}$. The set $\overline{\operatorname{conv}}(\mathcal{V})$ described in (34) is characterized by $g_{1}=-2, g_{2}=2$. For $\delta=1300$, the optimization problem (35) leads to a control law of the form (18) with:

$$
P=\left(\begin{array}{ccc}
0.026 & 1.78 .10^{-4} & 18.24 \\
1.78 .10^{-4} & 8.35 .10^{-5} & 0.24 \\
18.24 & 0.24 & 5.74 .10^{3}
\end{array}\right)
$$

The results are represented in Fig. 7 and Fig. 8. During the first $0.4 \mathrm{~ms}$, we can see that the control steers the state to the reference $V_{\text {ref }}$, and once the load variation occurs, it is observed that the steady state error is effectively cancelled and the perturbation rejected. In this case, the new equilibrium point is equal to : $\zeta_{\theta}^{*}=\left(\begin{array}{llll}2.4 & 12 & -0.0017\end{array}\right)^{\top}$. In [1], the authors deal with the perturbation rejection by assuming a bounded discrepancy in the modelling. However, its limits are not explicitly defined. Our method allows to guarantee the convergence of the controlled output to its reference for all unknown parameter which belong to a 

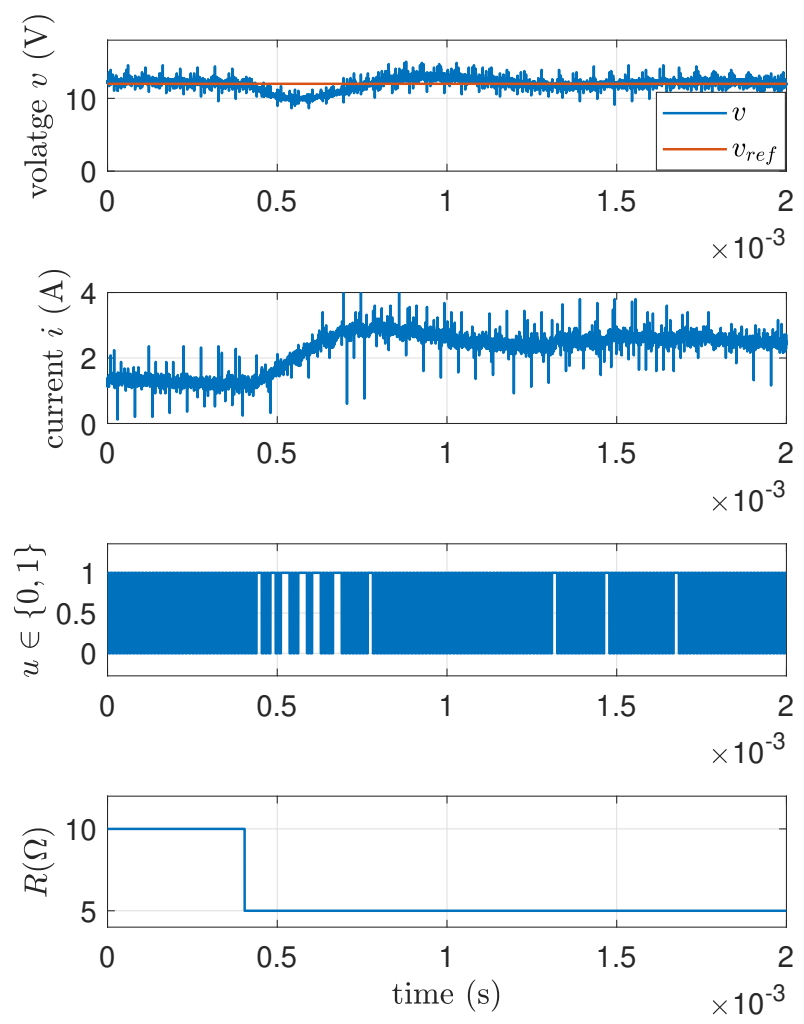

Fig. 7: Experimental results with integral action for relay control

known interval equal here to $[5,10] \Omega$.

\section{CONCLUSION}

In this paper, we have proposed an application of robust relay control applied to a buck converter. The design of the proposed control law uses the property of the existence of a linear state feedback and includes an integral action to cope with unknown load. A method capable of optimizing a subset included in the domain of attraction is presented. Experimental results show the effectiveness of the method which allows to cancel the steady state error and to reject load variations. Generalizing this approach for a larger class of switched system is the topic of future works.

\section{REFERENCES}

[1] G. Beneux, D. Astolfi, P. Riedinger, J. Daafouz, and L. Grimaud. Integral action for uncertain switched affine systems with application to DC/DC converters. 2018 European Control Conference (ECC), pages 795-800, June 2018.

[2] P. Bolzern and W. Spinelli. Quadratic stabilization of a switched affine system about a nonequilibrium point. In Proceedings of the 2004 American Control Conference, volume 5, pages 3890-3895. IEEE, 2004.

[3] G. S. Deaecto, J. C. Geromel, F. S. Garcia, and J. A. Pomilio. Switched affine systems control design with application to DC-DC converters. IET Control Theory Applications, 4(7):1201-1210, July 2010.
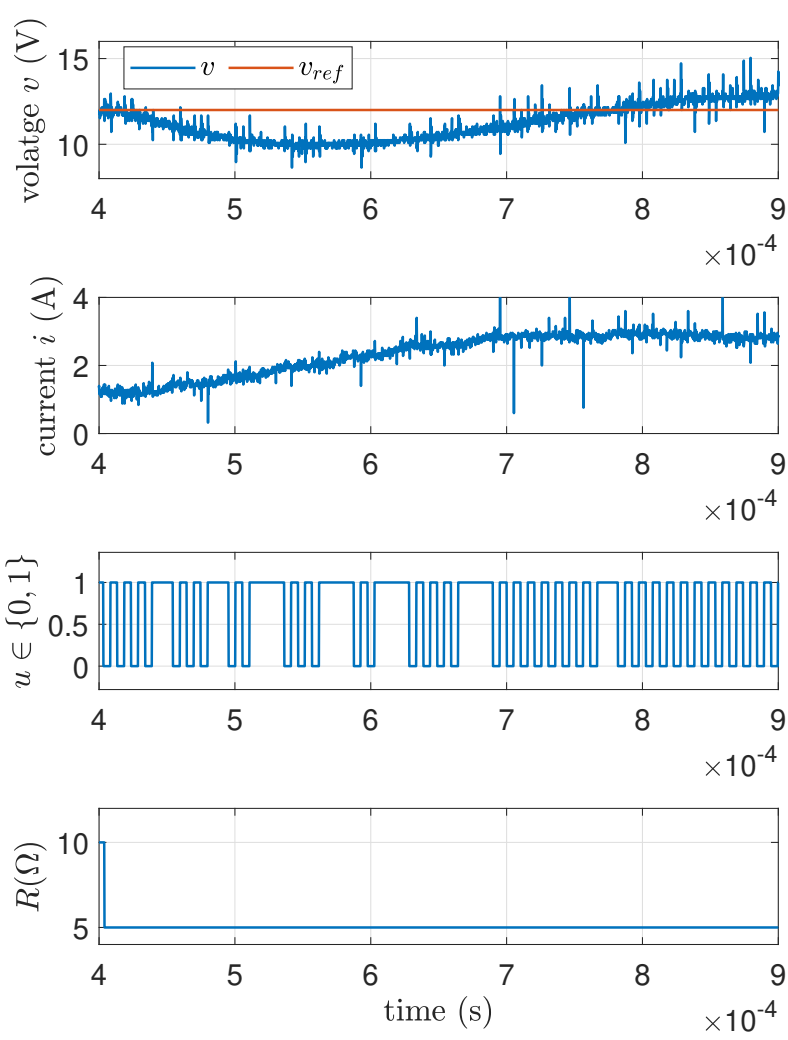

Fig. 8: Zoom of the figure 7

[4] R. A. Decarlo, M. S. Branicky, S. Pettersson, and B. Lennartson Perspectives and results on the stability and stabilizability of hybrid systems. Proceedings of the IEEE, 88(7):1069-1082, July 2000.

[5] R. W. ERICKSON. Fundamentals of Power electronics. Norwell, Massachusetts : Kluwer, 1999.

[6] A.-F. Filippov and F.-M. Arscott. Differential Equations with Discontinuous Righthand Sides: Control Systems. Mathematics and its Applications. Springer, 1988.

[7] L. Hetel, E. Fridman, and T. Floquet. Variable structure control with generalized relays: A simple convex optimization approach. IEEE Transactions on Automatic Control, 60(2):497-502, Feb 2015.

[8] M. K. Kazimierczuk. Pulse-width Modulated DC-DC Power Converters. John Wiley and Sons, 2008.

[9] D. Liberzon. Switching in systems and control. Birkhauser Basel, 2003.

[10] H. Lin and P. J. Antsaklis. Stability and stabilizability of switched linear systems: A survey of recent results. IEEE Transactions on Automatic Control, 54(2):308-322, Feb 2009.

[11] C. Olalla, I. Queinnec, R. Leyva, and A. El Aroudi. Optimal state-feedback control of bilinear dc-dc converters with guaranteed regions of stability. Industrial Electronics, IEEE Transactions on, 59(10):3868-3880, 2012.

[12] H. Sira-Ramirez and R. Silva-Ortigoza. Control Design Techniques in Power Electronis Devices. Springer, 2006.

[13] GE S.S. Sun Z. Switch linear system: control and design. Springer, 2005.

[14] A. Van der Shaft and H. Schumacher. An Introduction to Hybrid Dynamical Systems. Springer-Verlag London, 1999.

[15] G. Ziegler. Lectures on polytopes, volume 152. Springer Science \& Business Media, 2012. 